\title{
The Dialogue Dodecathlon: Open-Domain Knowledge and Image Grounded Conversational Agents
}

\author{
Kurt Shuster, Da Ju, Stephen Roller \\ Emily Dinan, Y-Lan Boureau, Jason Weston \\ Facebook AI Research \\ $\{$ kshuster, daju, roller, edinan, ylan, jase $\}$ efb.com
}

\begin{abstract}
We introduce dodecaDialogue: a set of 12 tasks that measures if a conversational agent can communicate engagingly with personality and empathy, ask questions, answer questions by utilizing knowledge resources, discuss topics and situations, and perceive and converse about images. By multi-tasking on such a broad large-scale set of data, we hope to both move towards and measure progress in producing a single unified agent that can perceive, reason and converse with humans in an open-domain setting. We show that such multi-tasking improves over a BERT pretrained baseline, largely due to multi-tasking with very large dialogue datasets in a similar domain, and that the multi-tasking in general provides gains to both text and image-based tasks using several metrics in both the finetune and task transfer settings. We obtain stateof-the-art results on many of the tasks, providing a strong baseline for this challenge.
\end{abstract}

\section{Introduction}

One of the goals of AI is to build a seeing, talking agent that can discuss, reason, empathize, and provide advice - in short a system that can perform natural communication displaying many of the properties expected when speaking to a human partner. Ideally, it should be able to be knowledgeable and personable, expert and engaging, serious or humorous - depending on the situation. It should be capable of answering questions, asking questions, responding to statements, having its own persona, and grounding the dialogue with external information and images.

While no single task exists that can train an agent or measure its ability on all of these axes at once, a number of distinct large-scale datasets targeting subsets of these skills have recently become available. We thus assemble these disparate tasks to form a single challenge: dodecaDialogue, consisting of 12 subtasks. Each contains both training data to build the skills we desire for our agent, and validation and test sets to measure our agent's ability at that skill. The overall goal is a single agent that can display all these skills. As some of the subtasks have very large datasets, e.g. 2.2 billion utterances, they can possibly help the agent with other skills too.

We thus build a model capable of training and multi-tasking on all these sources. We employ a transformer-based architecture (Vaswani et al., 2017) which accepts an image, external textual information and dialogue history as input, and generates a response for a given dialogue turn. Practically, by pre-training on the largest of the subtasks and then multi-tasking on all them, we can obtain state-of-the-art results compared to existing independently reported performance on all 10 of the 12 subtasks that have previous comparable results. We hence set a strong baseline for this challenge. While many existing approaches use large-scale pre-training on general text corpora, we show that using dialogue datasets instead, which are more closely linked to the desired agent's goals, is a strong alternative.

However, many challenges remain. While multitasking performs well, and has clear benefits, as shown in other works (Liu et al., 2015; Raffel et al., 2019), when compared to fine-tuning of the same system we do obtain typically small losses. Zeroshot transfer to left-out tasks is also demanding for current approaches. We analyze these aspects, along with our model's ability to ground on external knowledge and images in conjunction with the dialogue context, the impact of decoding algorithms, analysis of the weighting of tasks during multi-tasking as well as cross-task transfer ability in order to shed light and make progress on this challenging topic. 


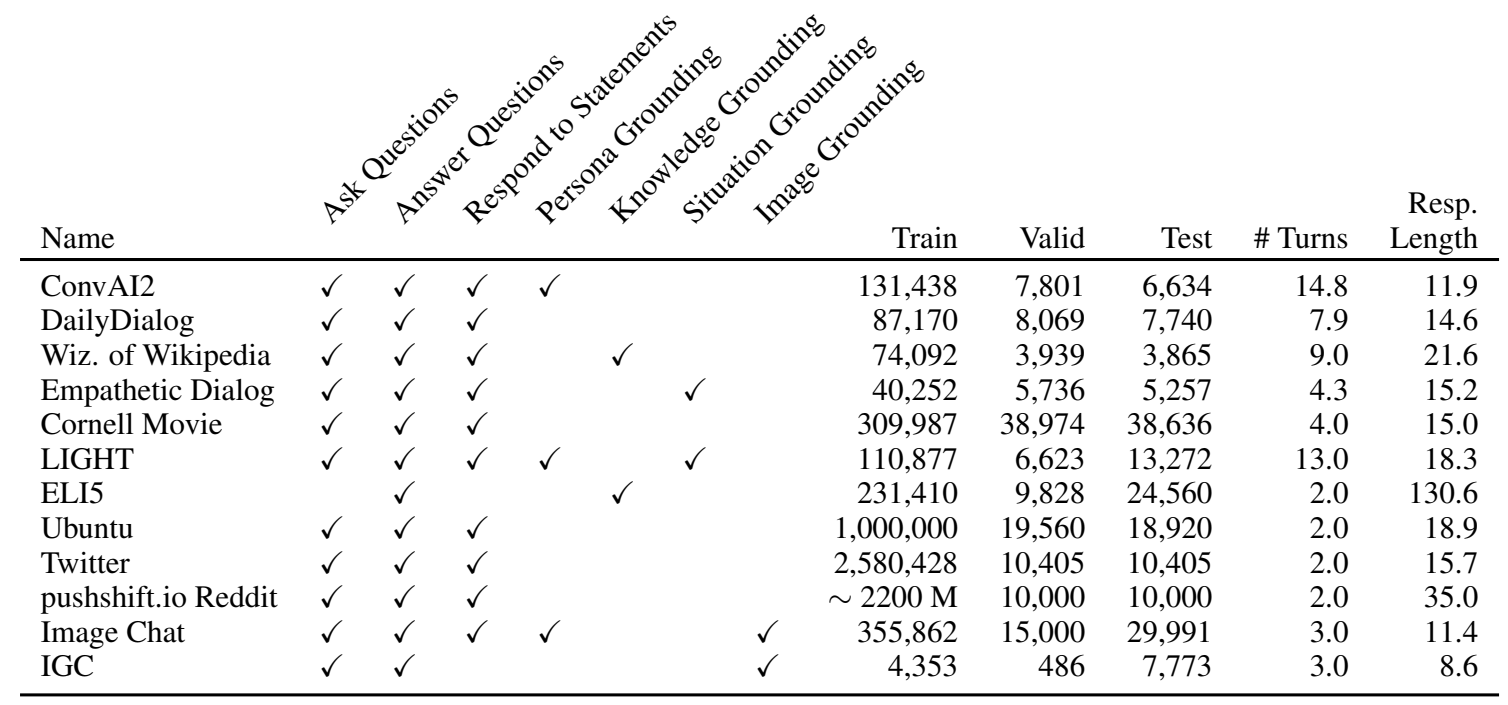

Table 1: The 12 dodecaDialogue subtasks, their sizes (number of train, valid, test utterances), and average number of turns and response length (words).

\section{The dodecaDialogue Task}

The dodecaDialogue task is intended to assemble important aspects of an engaging conversational agent into a single collection, where each subtask covers some of those goals. Such an agent should be able to get to know you when you first talk to it (ConvAI2), discuss everyday topics (DailyDialog, pushshift.io Reddit, Twitter, Cornell Movie), speak knowledgeably at depth (Wizard of Wikipedia, Ubuntu) and answer questions on such topics (ELI5). It must be able to handle situated conversations and demonstrate empathy (Empathetic Dialog, LIGHT) . It can also discuss images, as this is a vital part of human connection (Image Chat, IGC). We note that all of the provided subtasks are in English.

The overall statistics of the subtasks are given in Table 1. We now discuss each in turn.

ConvAI2 ConvAI2 is a dataset used at the NeurIPS 2018 competition of the same name, and is based on PersonaChat (Zhang et al., 2018; Dinan et al., 2020). The training data involves paired crowdworkers having a conversation where they get to know each other, in which each is given a role to play based on sentences describing their persona, which were also separately crowdsourced (while they cannot see their partner's persona). It thus involves asking and answering questions, responding in kind, and getting to know the other speaker and engaging them in friendly conversation - useful skills for an open-domain conversational agent.
DailyDialog Li et al. (2017) built a dialogue dataset intended to reflect conversations occurring in daily life. It covers ten categories ranging from holidays to financial topics, rather than focusing on one domain. Compared to ConvAI2, these conversations seem more in keeping with partners who already know each other, and want to discuss typical life details, again useful skills for a conversational agent. The dataset is also annotated with topic, emotion and utterance acts, but here we ignore these annotations and learn only from the utterances in the dialogue turns.

Wizard of Wikipedia This task involves discussing a given topic in depth, where the goal is to both engage the partner as well as display expert knowledge (Dinan et al., 2019). The training set consists of 1247 topics and a retrieval system over Wikipedia from which the dialogues were grounded during the human-human crowdsourced conversations. The topics were also crowdsourced and range from e-books to toga parties to showers. A model can thus learn to also perform similar retrieval and grounding at test time to potentially discuss any topic if it can generalize. We use the gold knowledge version of the task. We see this skill as a core component of an agent being able to not just chitchat, but actually engage a user in discussing real information about the world, e.g. by retrieving over documents from the internet.

Empathetic Dialogues Rashkin et al. (2019) constructed a dataset of crowdworker conversations grounded in an emotional situation. In each dia- 
logue, one speaker describes a personal situation and the other plays a "listener" role, displaying empathy during the discussion. The dataset contains descriptions of the situations being discussed with an attached emotion label, but these are not used here. Trained models are measured playing the part of the empathetic listener, an important feature of an agent to which humans wish to speak.

Cornell Movie Danescu-Niculescu-Mizil and Lee (2011) constructed a corpus containing a collection of fictional conversations from movie scripts, thus covering a large diversity of topics and emotional states.

LIGHT LIGHT (Urbanek et al., 2019) involves situated interactions between characters in a text adventure game. Similar to ConvAI2, personas for each character are given, with the training set including conversations between crowdworkers playing those roles. Different from ConvAI2, included are emotes and actions grounded within the game world (e.g. picking up and giving objects). As such, it measures the ability of a conversational agent to ground its discussion on a dynamic environment.

ELI5 ELI5 (Fan et al., 2019) involves long-form question answering grounded on multiple retrieved documents in order to answer common questions which people ask on the popular ELI5 subreddit. As such, the answers are in a conversational form applicable to a dialogue agent.

Ubuntu Lowe et al. (2015) built a dataset that involves in-depth discussions in solving Ubuntu problems. This studies the ability of an agent on a very focused single topic, and is also a standard benchmark in the field.

Twitter We use a variant of Twitter discussions (text-only), which have been used in many existing studies, e.g. Sordoni et al. (2015); See et al. (2019). This data naturally involves everyday discussions about topics that people care about. The public forum makes them different from the more personal discussions of some of the other tasks. This is the second largest dataset in the collection, and we thus measure in experiments its ability to help performance on other tasks.

pushshift.io Reddit We use a variant of Reddit discussions (text-only), which has also been used in several existing studies, see e.g. Yang et al. (2018); Mazaré et al. (2018); Keskar et al. (2019). Following Humeau et al. (2019), we use a previously existing Reddit dataset extracted and obtained by a third party and made available on pushshift.io, training to generate a comment conditioned on the full thread leading up to the comment, spanning $2200 \mathrm{M}$ training examples. This is the largest dataset in the collection - much larger than the others. The subreddits cover a vast range of topics, and hence this is a strong candidate for helping improve performance on other tasks via pre-training and multi-tasking. Note this dataset does not overlap with ELI5.

Image Chat Shuster et al. (2018) collected a crowdsourced dataset of human-human conversations about an image with a given personality, where the goal is to engage the other speaker. As such, it covers natural conversational responses, including displays of emotion and humor.

Image Grounded Conversations (IGC) IGC (Mostafazadeh et al., 2017) similarly involves two speakers discussing an image, here focusing on questions and responses. It only includes a validation and test set, and so we converted most of the validation set to form a small training set.

\subsection{Evaluation}

Metrics For all tasks, we use the following metrics: perplexity (PPL), BLEU, ROUGE-1,-2 and -L and F1, and also pick the metric most used in the literature as that subtask's 'Score' to compare to existing work.

Multi-tasking As we are interested in building a single conversational agent, we measure the ability of multi-tasked models that can perform all twelve tasks at once.

Single-Task Fine-tuning We can still compare such multi-tasked models to single-task fine-tuned baselines to assess if we have gained or lost performance. Like other works (Liu et al., 2015; Raffel et al., 2019) we also consider a multi-task followed by finetune setup in order to see if this produces better models. The latter tests if multi-tasking still proves useful in the single-task setting.

Zero-shot Transfer Finally, we consider a leaveone-out zero-shot setting whereby training is constrained to be on all the training data except for the task being evaluated. This evaluates the performance on truly new unseen tasks, an important behavior given there are always new tasks. 


\section{Related Work}

\subsection{Existing Models and Results}

Where possible, we have tried to track the best existing results for each task and provided a comparison in our final results table.

As ConvAI2 was a competition, a number of competitors built strong models on it. The best results were obtained by large pre-trained transformers (Dinan et al., 2020). In particular, Wolf et al. (2019b) pre-trained via the method of Radford et al. (2018) using the BooksCorpus dataset, resulting in the best perplexities and F1 scores. Since then, results have gotten even better with the advent of better and larger pretraining (Lewis et al., 2019), which we compare to here; the same work also reports strong results on ELI5.

He et al. (2019) recently obtained strong results on the DailyDialog and Cornell Movie tasks in terms of perplexity by pre-training on $10 \%$ of CCNEWS (Bakhtin et al., 2019), thus using 100 million sentences (2.7 billion words) and then finetuning a transformer based model with a multi-task strategy.

Overall, large pre-trained transformers indeed provide strong existing results on many of the tasks. Several large language modeling projects have been undertaken in order to show prowess in multi-tasking ability (Radford et al., 2019; Keskar et al., 2019), and transformer-based approaches have been adapted to language and vision tasks as well (Lu et al., 2019; Tan and Bansal, 2019; Li et al., 2019a; Shuster et al., 2018). As well as citing the relevant papers' results where possible in the experiments section, we also train a BERTbased (Devlin et al., 2019) generative model as an additional baseline.

\subsection{Related Tasks and Collections}

In the interests of feasibility, there are tasks we did not include in dodecaDialogue. For example, there are additional knowledge tasks (Qin et al., 2019; Moghe et al., 2018; Gopalakrishnan et al., 2019) and image-based datasets (Das et al., 2017) one could use. There are also a large number of QA tasks we did not include, e.g. Rajpurkar et al. (2016); Choi et al. (2018). In general, our choices were made based on tasks that after training might produce an engaging dialogue agent that humans naturally would want to talk to - which means either natural datasets or crowdsourced datasets where crowdworkers were encouraged to engage one another. As computational resources and ambitions scale, it would be interesting to add more tasks as well, while retaining the twelve we have chosen here in order to continue to evaluate their success, whilst extending the scope of the entire system.

All the subtasks in the collection we use here already exist. Other research projects have also built such collection-based tasks before as well. In particular, the NLP decathlon (McCann et al., 2018), from which the name of this paper is inspired, collects together a diverse set of NLP tasks - from sentiment detection to parsing. Talmor and Berant (2019) collect a set of 10 QA datasets and build MultiQA. Recently, (Raffel et al., 2019) also similarly multi-tasked a large set of NLP tasks, on an even bigger scale. Our work differs from these in that it is focused on dialogue tasks which naturally group together to form a conversational agent.

\section{Models}

BERT baseline. We implement a generative baseline using BERT via adapting the model using a standard auto-regressive loss. We concatenate both the context and current generation and provide these as input to the model, using BERT's sentence embeddings to distinguish the roles in the network. Although BERT is trained to predict masked tokens, we find that fine-tuning can easily adjust its behavior to predicting the next token. Our BERT baseline is roughly equivalent to the model of Wolf et al. (2019b), but does not have a classification loss term. The implementation relies on HuggingFace Transformers (Wolf et al., 2019a). We thus finetune this model for each of our tasks, except Image Chat and IGC which require images as input.

Image+Seq2Seq. We use a modification of a transformer Seq2Seq architecture (Vaswani et al., 2017), additionally adding image features to the encoder. Our model is a 8 layer encoder, 8 layer decoder with 512 dimensional embeddings and 16 attention heads, and is based on the ParlAI implementation (Miller et al., 2017). We use BPE following Humeau et al. (2019) via lower-cased Wikipedia, Toronto Books, and Open Subtitles with 30k merges, giving 54,940 terms. Reported perplexities are computed with this dictionary. For image features, we use the pre-trained image features from the ResNeXt-IG-3.5B model, a ResNeXt 32 $\mathrm{x} 48 \mathrm{~d}$ architecture (Xie et al., 2017) trained on 3.5 billion Instagram images following the procedure 


\begin{tabular}{|c|c|c|c|c|c|c|c|c|c|}
\hline & & & & & & & & & \\
\hline ConvAI2 & 19.4 & 43.3 & 38.9 & 28.7 & 18.3 & 11.4 & 11.2 & 11.3 & 16.4 \\
\hline DailyDialog & 15.2 & 37.8 & 32.8 & 20.8 & 18.2 & 10.4 & 10.2 & 11.8 & 15.5 \\
\hline Wiz. of Wikipedia & 14.1 & 40.7 & 36.0 & 37.3 & 15.3 & 8.7 & 8.5 & 8.7 & 13.2 \\
\hline Empathetic Dialog & 23.2 & 47.1 & 40.5 & 23.1 & 14.4 & 11.3 & 11.1 & 11.2 & 13.0 \\
\hline Cornell Movie & 29.4 & 46.2 & 44.8 & 34.2 & 27.8 & 20.0 & 19.8 & 22.3 & 25.4 \\
\hline LIGHT & 29.7 & 63.6 & 57.5 & 40.0 & 32.9 & 18.7 & 18.7 & 19.0 & 26.9 \\
\hline ELI5 & 28.1 & 62.9 & 58.8 & 63.8 & 31.2 & 21.2 & 21.1 & 25.0 & 31.1 \\
\hline Ubuntu & 20.7 & 35.8 & 34.5 & 38.5 & 31.1 & 17.3 & 17.2 & 23.3 & 30.8 \\
\hline Twitter & 37.0 & 61.9 & 59.3 & 59.3 & 53.6 & 29.8 & 29.8 & 37.0 & 52.8 \\
\hline pushshift.io Reddit & 39.0 & 27.8 & 27.8 & 27.8 & 27.8 & 27.8 & 25.8 & 28.0 & 106.3 \\
\hline Image Chat & N/A & 40.1 & 37.4 & 31.1 & 32.5 & 18.3 & 18.3 & 21.8 & 29.3 \\
\hline IGC & N/A & 86.3 & 79.5 & 23.1 & 14.6 & 10.0 & 10.0 & 10.2 & 12.2 \\
\hline dodecaScore & N/A & 49.5 & 45.7 & 35.6 & 26.5 & 17.1 & 16.8 & 19.1 & 31.1 \\
\hline
\end{tabular}

Table 2: Validation perplexity for the dodecaDialogue tasks in various settings.

described by Mahajan et al. (2018). This model was previously used successfully for the Image Chat task in Shuster et al. (2018). The final encoding from the ResNeXt model is a vector of size 2048; we then use a linear layer to project into the same size as the text encoding, and add it as an extra token at the end of the transformer's encoder output, then feed them all into the decoder. During fine-tuning we train the text transformer, but leave the image encoding fixed, apart from finetuning the linear projection. The text transformer is fine-tuned with a standard auto-regressive negative log-likelihood (NLL) loss, following usual sequence to sequence training schemes.

Our best models are available at https:// parl.ai/projects/dodecadialogue.

\section{Experiments}

Task Training We employ the ParlAI framework (Miller et al., 2017) for training on single tasks and for multi-tasking, as many of the tasks are already implemented there, along with a (multitask) training and evaluation framework for such models.

Pre-training As pushshift.io Reddit and (to some extent) Twitter are much larger than our other tasks, we try pre-training the Seq2Seq module of our Image+Seq2Seq networks with those datasets, before multi-tasking on all of the tasks, or for evaluating single task fine-tuning.

For Reddit, the model was trained to generate

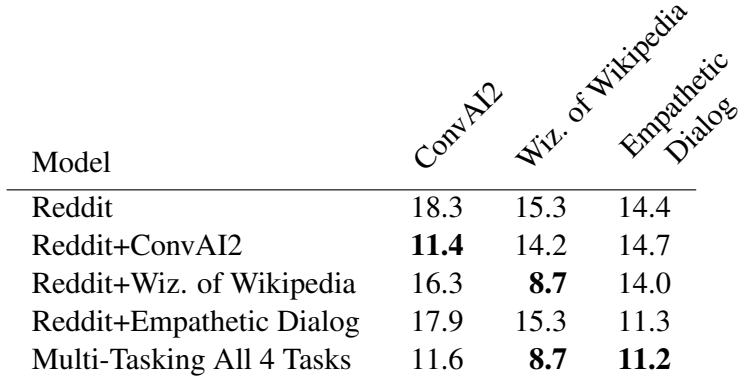

Table 3: Transfer performance of various multi-task models (validation perplexity).

a comment conditioned on the full thread leading up to the comment. Comments containing URLs or that were under 5 characters in length were removed from the corpus, as were all child comments. Comments were truncated to $1024 \mathrm{BPE}$ tokens. The model was trained with a batch size of 3072 sequences for approximately $3 \mathrm{M}$ updates using a learning rate of $5 \mathrm{e}-4$, and an inverse square root scheduler. This took approximately two weeks using 64 NVIDIA V100s. We note that our transformer pre-training only includes text, while our image encoder was pre-trained separately in previous work (Mahajan et al., 2018). Learning how to combine these sources occurs during fine-tuning.

It is important to note that, while compute-heavy, pre-training was conducted exactly once, and all of the subsequent fine-tuning is significantly faster to run. 


\begin{tabular}{lrr} 
Knowledge grounding & Without & With \\
Wiz. of Wikipedia & 16.8 & $\mathbf{8 . 7}$ \\
ELI5 & 21.3 & $\mathbf{2 1 . 2}$ \\
\hline Image grounding & & \\
Image Chat & 19.5 & $\mathbf{1 8 . 3}$ \\
IGC & $\mathbf{1 0 . 1}$ & $\mathbf{1 0 . 1}$
\end{tabular}

Table 4: The impact of knowledge and image grounding in dodecaDialogue (validation perplexity).

Transfer Performance between Tasks We first perform a preliminary study on a subset of the tasks: Reddit, ConvAI2, Wizard of Wikipedia and Empathetic Dialogues, and report the transfer ability of training on some of them, and testing on all of them (using the validation set), reporting perplexity. The results are reported in Table 3. They show that training on pushshift.io Reddit alone, a huge dataset, is effective at transfer to other tasks, but never as effective as fine-tuning on the task itself. Moreover, fine-tuning on most of the smaller tasks actually provides improvements over pushshift.io Reddit training alone at transfer, likely because the three tasks selected are more similar to each other than to pushshift.io Reddit. Finally, training on all four tasks is the most effective strategy averaged over all tasks compared to any other single model, although this does not beat switching between different fine-tuned models on a per-task basis.

\section{Comparison of Pre-training + Fine-tuning} strategies Across all 12 tasks, we compare several pre-training strategies: using BERT, no pretraining at all, only initializing via fastText (Joulin et al., 2017), and using Twitter and pushshift.io Reddit pre-training with our Image+Seq2Seq architecture. For each variant we tune the learning rate, layers, number of heads and embedding size, with less pre-training typically requiring smaller capacity models. We then only fine-tune on a single task in these experiments, and report perplexity for that task alone, over all 12 tasks. The results are given in Table 2, reporting results on the validation set ${ }^{1}$.

The results show a clear reduction in perplexity with more pre-training, as expected. This is most easily seen by the dodecaScore (last row) that is the mean perplexity over all 12 tasks, which decreases from 49.5 (from scratch models) down to 17.1 with pushshift.io Reddit pre-training. FastText (45.7) and Twitter (35.6) initializations help, but nowhere near as much. BERT fares better, but still is clearly

\footnotetext{
${ }^{1}$ We choose not to use the test set here as we report so many numbers, we do not want to overuse it.
}

\begin{tabular}{l|rrrrrrr} 
& \multicolumn{8}{|c}{ Relative Task Weighting } \\
& 1 & 2 & 5 & 10 & 20 & 50 & $\infty$ \\
Cornell & 21.9 & 21.5 & 20.6 & 20.1 & 19.9 & $\mathbf{1 9 . 8}$ & - \\
Fine-tuned & 20.1 & 20.0 & 20.0 & 19.9 & $\mathbf{1 9 . 8}$ & $\mathbf{1 9 . 8}$ & 20.0 \\
\hline ELI5 & 25.0 & 24.1 & 22.8 & 22.2 & 21.6 & 21.3 & - \\
Fine-tuned & 21.8 & 21.6 & 21.4 & 21.3 & $\mathbf{2 1 . 1}$ & $\mathbf{2 1 . 1}$ & 21.2 \\
\hline Ubuntu & 23.1 & 22.2 & 20.6 & 19.6 & 18.6 & 17.4 & - \\
Fine-tuned & 18.2 & 18.1 & 17.8 & 17.7 & $\mathbf{1 7 . 2}$ & $\mathbf{1 7 . 2}$ & 17.3
\end{tabular}

Table 5: Validation perplexity on select $d o$ decaDialogue tasks comparing relative weights of tasks during multi-tasking, followed by fine-tuning (row below). The relative task weight is the ratio of examples from that task compared to others presented during multitasking. $\infty$ indicates single-task training.

\begin{tabular}{|c|c|c|c|c|c|c|}
\hline \multirow[b]{3}{*}{ Task } & \multicolumn{6}{|c|}{ N-gram } \\
\hline & & Bean & Size & & Block & Nucleus \\
\hline & 1 & 2 & 3 & 5 & $N=3$ & $p=0.3$ \\
\hline ConvAI2 & 20.0 & 21.0 & 21.3 & 21.2 & 21.3 & 18.7 \\
\hline WoW & 35.9 & 37.4 & 37.8 & 37.9 & 37.9 & 31.1 \\
\hline
\end{tabular}

Table 6: Impact of the decoding strategy on select tasks, reporting validation F1 score for the All Tasks MT model. N-gram block is for best beam size.

worse than pushshift.io Reddit pre-training. The hypothesis here is that pushshift.io Reddit yields much more effective transfer as it is a dialogue task like our others, whereas non-dialogue corpora such as Wikipedia are not. This was previously observed for retrieval models in Humeau et al. (2019). Note that we do not report results for the image dialogue tasks for BERT as that architecture does not deal with images.

Finally, as pushshift.io Reddit is so effective, we also compare to pushshift.io Reddit training only, with no fine-tuning at all across all tasks, similar to our initial study in Table 3. The performance is impressive, with some tasks yielding lower perplexity than BERT pre-training + single task finetuning. However, it still lags significantly behind fine-tuning applied after pushshift.io Reddit pretraining.

Image and Knowledge Grounding Some of our tasks involve grounding on knowledge or images. To show such grounding helps, we report results with and without grounding on those tasks in Table 4, reporting perplexity. Particularly for Wizard of Wikipedia (knowledge) and Image Chat (images) such grounding has a clear effect.

Multi-Task Results Next, we perform multitask training across all tasks, which is our ultimate goal in order to obtain an open-domain conversational agent. We optimize over the same set of 


\begin{tabular}{|c|c|c|c|c|c|c|c|c|}
\hline \multirow[b]{3}{*}{ ConvAI2 } & \multicolumn{4}{|c|}{ Existing Approaches (independent) } & \multicolumn{2}{|c|}{$\mathrm{MT}+\mathrm{FT}$} & \multicolumn{2}{|c|}{ All Tasks MT } \\
\hline & Approach & PPL & Score & (Metric) & PPL & Score & PPL & Score \\
\hline & (Lewis et al., 2019) & *11.9 & $* 20.7$ & F1 & 11.1 & 21.6 & 10.8 & 21.7 \\
\hline DailyDialog & (He et al., 2019) & 11.1 & - & F1 & 10.4 & 18.2 & 12.0 & 16.2 \\
\hline Wiz. of Wikipedia & (Dinan et al., 2019) & 23.1 & 35.5 & F1 & 8.3 & 38.4 & 8.4 & 38.4 \\
\hline Empathetic Dialog & (Rashkin et al., 2019) & 21.2 & 6.27 & Avg-BLEU & 11.4 & 8.1 & 11.5 & 8.4 \\
\hline Cornell Movie & (He et al., 2019) & 27.5 & - & F1 & 20.2 & 12.4 & 22.2 & 11.9 \\
\hline LIGHT & (Urbanek et al., 2019) & *27.1 & ${ }^{*} 13.9$ & F1 & 18.9 & 16.2 & 19.3 & 16.1 \\
\hline ELI5 & (Lewis et al., 2019) & 24.2 & 20.4 & Avg-ROUGE & 21.0 & 22.6 & 24.9 & 20.7 \\
\hline Ubuntu & (Luan et al., 2016) & 46.8 & - & $\mathrm{F} 1$ & 17.1 & 12.7 & 23.1 & 12.1 \\
\hline Twitter & & - & - & F1 & 30.7 & 9.9 & 38.2 & 9.8 \\
\hline pushshift.io Reddit & & - & - & F1 & 25.6 & 13.6 & 27.8 & 13.5 \\
\hline Image Chat & (Shuster et al., 2018) & - & 27.4 & ROUGE-L $\left(1^{s t}\right.$ turn $)$ & 18.8 & 43.8 & 22.3 & 39.7 \\
\hline IGC & (Mostafazadeh et al., 2017) & - & 1.57 & BLEU (responses) & 11.9 & 9.9 & 12.0 & 8.2 \\
\hline
\end{tabular}

Table 7: Test performance for various metrics on the dodecaDialogue tasks comparing our multi-task and multitask + fine-tuned methods to existing approaches (cited). Dashes mean metric was not provided. ${ }^{*}$ was reported on validation only. Score is defined on a per-task basis in the metric column.

hyperparameters as before, including multi-tasking weights for tasks, where one samples during training with differing probabilities, and we choose the best model by performing early stopping on the average performance across all tasks. In this way, we treat all 12 tasks as a single task, and thus during test time it is the model's responsibility to understand how to respond from the context (image/dialogue) itself.

In the end we did not obtain clear improvements beyond pre-training with pushshift.io Reddit and then equally sampling from all tasks. We report that final model's validation performance in terms of perplexity in Table 2 (second to last column, “All Tasks MT"). It achieves a dodecaScore of 19.1, superior to all pre-train fine-tune approaches except pushshift.io Reddit pre-training followed by finetuning, and is also superior to a single pushshift.io Reddit model. However, comparing across tasks, while most are close to the corresponding best finetuned model, many are just slightly worse. This is an expected result and is often reported in multitask systems (Raffel et al., 2019). We look upon this result as both positive - we can obtain a single model doing well on all tasks, which a fine-tuned model cannot - whilst also remaining a challenge to the community: can one find architectures that leverage multi-tasking even better?

Multi-Task followed by Fine-Tuning As also performed in Liu et al. (2015); Raffel et al. (2019) we can try to train in a multi-task manner on all tasks, before fine-tuning on a single task, and build a separate model performing this procedure for all tasks, in an attempt to improve single task results further. Using this approach, one is free to perform hyperparameter search differently for each task. Here, we found that applying relative task up-weighting during multi-tasking training made a clear difference to the final quality of the fine-tuned target task model, see Table 5. Generally, better results come from assigning most of the multi-task weight towards the task itself to be fine-tuned. Using such an approach we can get marginally better results than fine-tuning alone, although the differences are generally small. The final best models per task are shown compared to other approaches in Table 2 (third to last column, "MT All Tasks + FT Single Task"). The final validation dodecaScore is 16.8 , only slightly below 17.1 for fine-tuning.

Decoding Strategies So far, we have only been measuring perplexity, but we are actually interested in generation, which requires us to decode. We consider several standard approaches: greedy, beam search (with beam size, and minimum and maximum output length ${ }^{2}$ hyperparameters), beam search with beam blocking (blocking $n$-grams, we use $n=3$ ) (Paulus et al., 2018) and nucleus sampling (with parameter $p$ ) (Holtzman et al., 2019). We show the effect of these choices in Table 6 for ConvAI2 and Wizard of Wikipedia (WoW).

Final Systems The final test performance for our best multi-task and fine-tuned (via multi-task followed by fine-tuning) systems are reported in Table 7 (right), with more detailed results with all decoding-based metrics, and validation as well as test performance in Appendix A. Here, for the multi-task model we have fine-tuned the decoding hyperparameters per task. For results with a single set of decoding hyperparameters, see also

\footnotetext{
${ }^{2}$ The length parameters are important for ELI5.
} 
Appendix A. We generally find across all metrics a similar story as before when comparing the finetuning with multi-tasking: multi-tasking is successful, but the challenge is still to do better.

Comparison to Existing Systems We compare to existing state-of-the-art results previously published for each task. Results are given in Table 7. As existing works report different metrics per task, we report perplexity where possible (but note, they may be computed on a different dictionary), and choose the sequence decoding-based metric that is commonly reported per task (listed in column 'Metric'), where the 'Score' column reports its value. We compare these to our best fine-tuned and multitasked models. Our multi-task model outperforms all available existing results, with 2 of the 12 tasks having no previous result. It is only surpassed by our fine-tuned model which also outperforms all available existing results. Overall, our methods set a strong challenge to future approaches.

Human Evaluation In addition to automatic metrics, we perform human evaluation on two of the tasks to assess the abilities of our All Tasks MT conversational agent: the knowledge grounding task Wizard of Wikipedia (WoW) and the image grounding task Image Chat. We follow the same evaluation protocols as in Dinan et al. (2019); Shuster et al. (2018), comparing our method to the existing approaches referenced in Table 7. This involves collecting 100 human-bot conversations for WoW using crowdworkers, involving 8-10 turns each, across seen topics (seen in the training set) and unseen topics, and 500 image-based responses for Image Chat. A separate set of crowdworkers are then used to compare models pairwise following the ACUTE-Eval procedure of (Li et al., 2019b), where they are asked to choose which is "the more engaging response" for Image Chat (1500 trials) and "Who would you prefer to talk to for a long conversation?" for WoW (400 trials).

The results, given in Figure 1, show our method outperforming the existing state of the art generative models on all three comparisons: Image Chat, WoW seen topics and WoW unseen topics. All three results are statistically significant (binomial test, $p<.05$ ). Additional details and results breakdown are given in Appendix Section B.

Example Outputs We show some example outputs of our multi-task model for some of the tasks in Appendix C. Our model is able to leverage im-

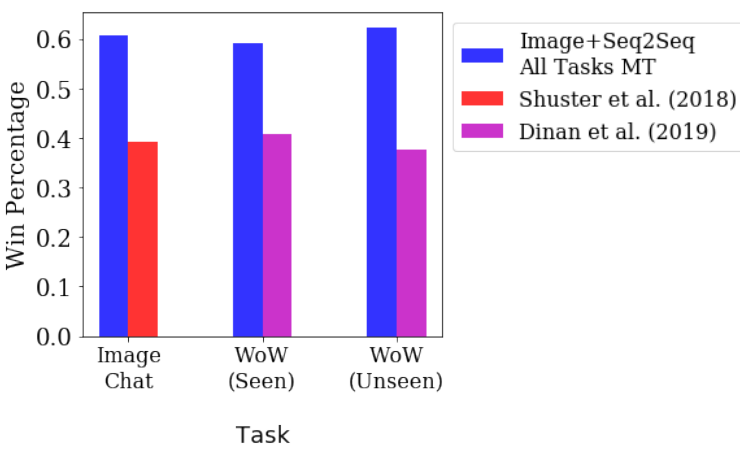

Figure 1: Human evaluations on Image Chat and Wizard of Wikipedia (WoW), comparing existing state of the art models with our All Tasks MT conversational agent. Engagingness win rates are statistically significant in all three matchups (binomial test, $p<.05$ ).

ages, knowledge, and given personality attributes to produce engaging dialogue with a large amount of variety, depending on the situation.

Leave-One-Out Zero-Shot Performance Last, but not least, we evaluate the performance of a multi-task model at zero-shot transfer to a new dialogue task. This is performed by training on all but one of the tasks, and reporting performance on the left out one, repeating this experiment for all tasks. Our best performing models in that regard are reported in Table 2 (last column). First, it is reassuring that the overall scores are reasonable, outperforming a pushshift.io Reddit only model on every task except pushshift.io Reddit itself. This means that multi-tasking across many tasks helps transfer learning. However, the gap between zeroshot performance and multi-task or fine-tuning performance means there is still a significant challenge in improving these results. Finally, we believe that reporting results in this regime in addition to multitasking results may help avoid the temptation to "cheat" at multi-tasking by trying to detect the task and then apply a separate fine-tuned classifier, as presumably that approach will not truly leverage reasoning and skills between tasks, which transfer may help measure.

\section{Discussion}

We have introduced the dodecaDialogue task, and provide strong baseline results leveraging multimodal Image+Seq2Seq transformers trained across all tasks. The goal of introducing this task is not just as another challenge dataset, but to further motivate building and evaluating conversational 
agents capable of multiple skills - one of the core goals of AI. We believe current systems are closer to that goal than ever before - but we also still have a long way to go.

Recently reported results show systems can be reasonably competitive compared to humans in particular domains for short conversations ( $\mathrm{Li}$ et al., 2019b; Shuster et al., 2018). This work tries to bridge the gap to avoid agents with niche skills, to move towards evaluating an open-domain set of skills. Still, despite leveraging 12 tasks, there are many skills not included in our set. For example, longer conversations involving memory (Moon et al., 2019), or mixing open-domain conversation with task oriented goals. Future work should consider adding these tasks to the ones used here, while continuing the quest for improved models.

\section{References}

Anton Bakhtin, Sam Gross, Myle Ott, Yuntian Deng, Marc'Aurelio Ranzato, and Arthur Szlam. 2019. Real or fake? learning to discriminate machine from human generated text. arXiv preprint arXiv:1906.03351.

Eunsol Choi, He He, Mohit Iyyer, Mark Yatskar, Wentau Yih, Yejin Choi, Percy Liang, and Luke Zettlemoyer. 2018. QuAC: Question answering in context. In Proceedings of the 2018 Conference on Empirical Methods in Natural Language Processing, pages 2174-2184, Brussels, Belgium. Association for Computational Linguistics.

Cristian Danescu-Niculescu-Mizil and Lillian Lee. 2011. Chameleons in imagined conversations: A new approach to understanding coordination of linguistic style in dialogs. In Proceedings of the 2nd Workshop on Cognitive Modeling and Computational Linguistics, Portland, Oregon, USA. Association for Computational Linguistics.

Abhishek Das, Satwik Kottur, Khushi Gupta, Avi Singh, Deshraj Yadav, José MF Moura, Devi Parikh, and Dhruv Batra. 2017. Visual dialog. In Proceedings of the IEEE Conference on Computer Vision and Pattern Recognition, pages 326-335.

Jacob Devlin, Ming-Wei Chang, Kenton Lee, and Kristina Toutanova. 2019. BERT: Pre-training of deep bidirectional transformers for language understanding. In Proceedings of the 2019 Conference of the North American Chapter of the Association for Computational Linguistics: Human Language Technologies, Volume 1 (Long and Short Papers), pages 4171-4186, Minneapolis, Minnesota. Association for Computational Linguistics.

Emily Dinan, Varvara Logacheva, Valentin Malykh, Alexander Miller, Kurt Shuster, Jack Urbanek, Douwe Kiela, Arthur Szlam, Iulian Serban,
Ryan Lowe, Shrimai Prabhumoye, Alan W. Black, Alexander Rudnicky, Jason Williams, Joelle Pineau, Mikhail Burtsev, and Jason Weston. 2020. The second conversational intelligence challenge (ConvAI2). In The NeurIPS '18 Competition, pages 187208, Cham. Springer International Publishing.

Emily Dinan, Stephen Roller, Kurt Shuster, Angela Fan, Michael Auli, and Jason Weston. 2019. Wizard of wikipedia: Knowledge-powered conversational agents. In Proceedings of the International Conference on Learning Representations.

Angela Fan, Yacine Jernite, Ethan Perez, David Grangier, Jason Weston, and Michael Auli. 2019. ELI5: Long form question answering. In Proceedings of the 57th Annual Meeting of the Association for Computational Linguistics, pages 3558-3567, Florence, Italy. Association for Computational Linguistics.

Karthik Gopalakrishnan, Behnam Hedayatnia, Qinlang Chen, Anna Gottardi, Sanjeev Kwatra, Anu Venkatesh, Raefer Gabriel, and Dilek HakkaniTür. 2019. Topical-Chat: Towards KnowledgeGrounded Open-Domain Conversations. In Proc. Interspeech 2019, pages 1891-1895.

Tianxing He, Jun Liu, Kyunghyun Cho, Myle Ott, Bing Liu, James Glass, and Fuchun Peng. 2019. Mixreview: Alleviate forgetting in the pretrain-finetune framework for neural language generation models. arXiv preprint arXiv:1910.07117.

Ari Holtzman, Jan Buys, Maxwell Forbes, and Yejin Choi. 2019. The curious case of neural text degeneration. arXiv preprint arXiv:1904.09751.

Samuel Humeau, Kurt Shuster, Marie-Anne Lachaux, and Jason Weston. 2019. Poly-encoders: Transformer architectures and pre-training strategies for fast and accurate multi-sentence scoring. arXiv preprint arXiv:1905.01969.

Armand Joulin, Edouard Grave, Piotr Bojanowski, and Tomas Mikolov. 2017. Bag of tricks for efficient text classification. In Proceedings of the 15th Conference of the European Chapter of the Association for Computational Linguistics: Volume 2, Short Papers, pages 427-431, Valencia, Spain. Association for Computational Linguistics.

Nitish Shirish Keskar, Bryan McCann, Lav R Varshney, Caiming Xiong, and Richard Socher. 2019. CTRL: A conditional transformer language model for controllable generation. arXiv preprint arXiv:1909.05858.

Mike Lewis, Yinhan Liu, Naman Goyal, Marjan Ghazvininejad, Abdelrahman Mohamed, Omer Levy, Ves Stoyanov, and Luke Zettlemoyer. 2019. BART: Denoising sequence-to-sequence pre-training for natural language generation, translation, and comprehension. arXiv preprint arXiv:1910.13461. 
Liunian Harold Li, Mark Yatskar, Da Yin, Cho-Jui Hsieh, and Kai-Wei Chang. 2019a. VisualBERT: A simple and performant baseline for vision and language. arXiv preprint arXiv:1908.03557.

Margaret Li, Jason Weston, and Stephen Roller. 2019b. ACUTE-EVAL: Improved dialogue evaluation with optimized questions and multi-turn comparisons. In Proceedings of the NeurIPS Workshop on Conversational AI.

Yanran Li, Hui Su, Xiaoyu Shen, Wenjie Li, Ziqiang Cao, and Shuzi Niu. 2017. DailyDialog: A manually labelled multi-turn dialogue dataset. In Proceedings of the Eighth International Joint Conference on Natural Language Processing (Volume 1: Long Papers), pages 986-995, Taipei, Taiwan. Asian Federation of Natural Language Processing.

Xiaodong Liu, Jianfeng Gao, Xiaodong He, Li Deng, Kevin Duh, and Ye-yi Wang. 2015. Representation learning using multi-task deep neural networks for semantic classification and information retrieval. In Proceedings of the 2015 Conference of the North American Chapter of the Association for Computational Linguistics: Human Language Technologies, pages 912-921, Denver, Colorado. Association for Computational Linguistics.

Ryan Lowe, Nissan Pow, Iulian Serban, and Joelle Pineau. 2015. The Ubuntu dialogue corpus: A large dataset for research in unstructured multi-turn dialogue systems. In Proceedings of the 16th Annual Meeting of the Special Interest Group on Discourse and Dialogue, pages 285-294, Prague, Czech Republic. Association for Computational Linguistics.

Jiasen Lu, Dhruv Batra, Devi Parikh, and Stefan Lee. 2019. ViLBERT: Pretraining task-agnostic visiolinguistic representations for vision-and-language tasks. arXiv preprint arXiv:1908.02265.

Yi Luan, Yangfeng Ji, and Mari Ostendorf. 2016. LSTM based conversation models. arXiv preprint arXiv:1603.09457.

Dhruv Mahajan, Ross Girshick, Vignesh Ramanathan, Kaiming He, Manohar Paluri, Yixuan Li, Ashwin Bharambe, and Laurens van der Maaten. 2018. Exploring the limits of weakly supervised pretraining. In Proceedings of the European Conference on Computer Vision, pages 185-201, Cham. Springer International Publishing.

Pierre-Emmanuel Mazaré, Samuel Humeau, Martin Raison, and Antoine Bordes. 2018. Training millions of personalized dialogue agents. In Proceedings of the 2018 Conference on Empirical Methods in Natural Language Processing, pages 2775-2779, Brussels, Belgium. Association for Computational Linguistics.

Bryan McCann, Nitish Shirish Keskar, Caiming Xiong, and Richard Socher. 2018. The natural language decathlon: Multitask learning as question answering. arXiv preprint arXiv:1806.08730.
Alexander Miller, Will Feng, Dhruv Batra, Antoine Bordes, Adam Fisch, Jiasen Lu, Devi Parikh, and Jason Weston. 2017. ParlAI: A dialog research software platform. In Proceedings of the 2017 Conference on Empirical Methods in Natural Language Processing: System Demonstrations, pages 79-84, Copenhagen, Denmark. Association for Computational Linguistics.

Nikita Moghe, Siddhartha Arora, Suman Banerjee, and Mitesh M. Khapra. 2018. Towards exploiting background knowledge for building conversation systems. In Proceedings of the 2018 Conference on Empirical Methods in Natural Language Processing, pages 2322-2332, Brussels, Belgium. Association for Computational Linguistics.

Seungwhan Moon, Pararth Shah, Rajen Subba, and Anuj Kumar. 2019. Memory grounded conversational reasoning. In Proceedings of the 2019 Conference on Empirical Methods in Natural Language Processing and the 9th International Joint Conference on Natural Language Processing (EMNLPIJCNLP): System Demonstrations, pages 145-150, Hong Kong, China. Association for Computational Linguistics.

Nasrin Mostafazadeh, Chris Brockett, Bill Dolan, Michel Galley, Jianfeng Gao, Georgios Spithourakis, and Lucy Vanderwende. 2017. Image-grounded conversations: Multimodal context for natural question and response generation. In Proceedings of the Eighth International Joint Conference on Natural Language Processing (Volume 1: Long Papers), pages 462-472, Taipei, Taiwan. Asian Federation of Natural Language Processing.

Romain Paulus, Caiming Xiong, and Richard Socher. 2018. A deep reinforced model for abstractive summarization. In Proceedings of the International Conference on Learning Representations.

Lianhui Qin, Michel Galley, Chris Brockett, Xiaodong Liu, Xiang Gao, Bill Dolan, Yejin Choi, and Jianfeng Gao. 2019. Conversing by reading: Contentful neural conversation with on-demand machine reading. In Proceedings of the 57th Annual Meeting of the Association for Computational Linguistics, pages 5427-5436, Florence, Italy. Association for Computational Linguistics.

Alec Radford, Karthik Narasimhan, Tim Salimans, and Ilya Sutskever. 2018. Improving language understanding by generative pre-training.

Alec Radford, Jeffrey Wu, Rewon Child, David Luan, Dario Amodei, and Ilya Sutskever. 2019. Language models are unsupervised multitask learners. OpenAI Blog, 1(8).

Colin Raffel, Noam Shazeer, Adam Roberts, Katherine Lee, Sharan Narang, Michael Matena, Yanqi Zhou, Wei Li, and Peter J Liu. 2019. Exploring the limits of transfer learning with a unified text-to-text transformer. arXiv preprint arXiv:1910.10683. 
Pranav Rajpurkar, Jian Zhang, Konstantin Lopyrev, and Percy Liang. 2016. SQuAD: 100,000+ questions for machine comprehension of text. In Proceedings of the 2016 Conference on Empirical Methods in Natural Language Processing, pages 2383-2392, Austin, Texas. Association for Computational Linguistics.

Hannah Rashkin, Eric Michael Smith, Margaret Li, and Y-Lan Boureau. 2019. Towards empathetic opendomain conversation models: A new benchmark and dataset. In Proceedings of the 57th Annual Meeting of the Association for Computational Linguistics, pages 5370-5381, Florence, Italy. Association for Computational Linguistics.

Abigail See, Stephen Roller, Douwe Kiela, and Jason Weston. 2019. What makes a good conversation? how controllable attributes affect human judgments. In Proceedings of the 2019 Conference of the North American Chapter of the Association for Computational Linguistics: Human Language Technologies, Volume 1 (Long and Short Papers), pages 1702-1723, Minneapolis, Minnesota. Association for Computational Linguistics.

Kurt Shuster, Samuel Humeau, Antoine Bordes, and Jason Weston. 2018. Engaging image chat: Modeling personality in grounded dialogue. arXiv preprint arXiv:1811.00945.

Alessandro Sordoni, Michel Galley, Michael Auli, Chris Brockett, Yangfeng Ji, Margaret Mitchell, Jian-Yun Nie, Jianfeng Gao, and Bill Dolan. 2015. A neural network approach to context-sensitive generation of conversational responses. In Proceedings of the 2015 Conference of the North American Chapter of the Association for Computational Linguistics: Human Language Technologies, pages 196205, Denver, Colorado. Association for Computational Linguistics.

Alon Talmor and Jonathan Berant. 2019. MultiQA: An empirical investigation of generalization and transfer in reading comprehension. In Proceedings of the 57th Annual Meeting of the Association for Computational Linguistics, pages 4911-4921, Florence, Italy. Association for Computational Linguistics.

Hao Tan and Mohit Bansal. 2019. LXMERT: Learning cross-modality encoder representations from transformers. In Proceedings of the 2019 Conference on Empirical Methods in Natural Language Processing and the 9th International Joint Conference on Natural Language Processing (EMNLP-IJCNLP), pages 5099-5110, Hong Kong, China. Association for Computational Linguistics.

Jack Urbanek, Angela Fan, Siddharth Karamcheti, Saachi Jain, Samuel Humeau, Emily Dinan, Tim Rocktäschel, Douwe Kiela, Arthur Szlam, and Jason Weston. 2019. Learning to speak and act in a fantasy text adventure game. In Proceedings of the 2019 Conference on Empirical Methods in Natural Language Processing and the 9th International Joint Conference on Natural Language Processing (EMNLP-IJCNLP), pages 673-683, Hong
Kong, China. Association for Computational Linguistics.

Ashish Vaswani, Noam Shazeer, Niki Parmar, Jakob Uszkoreit, Llion Jones, Aidan N Gomez, Ł ukasz Kaiser, and Illia Polosukhin. 2017. Attention is all you need. In Advances in Neural Information Processing Systems 30, pages 5998-6008. Curran Associates, Inc.

Thomas Wolf, Lysandre Debut, Victor Sanh, Julien Chaumond, Clement Delangue, Anthony Moi, Pierric Cistac, Tim Rault, Rémi Louf, Morgan Funtowicz, and Jamie Brew. 2019a. HuggingFace's transformers: State-of-the-art natural language processing. arXiv preprint arXiv:1910.03771.

Thomas Wolf, Victor Sanh, Julien Chaumond, and Clement Delangue. 2019b. TransferTransfo: A transfer learning approach for neural network based conversational agents. arXiv preprint arXiv:1901.08149.

Saining Xie, Ross Girshick, Piotr Dollár, Zhuowen Tu, and Kaiming He. 2017. Aggregated residual transformations for deep neural networks. In Computer Vision and Pattern Recognition (CVPR).

Yinfei Yang, Steve Yuan, Daniel Cer, Sheng-yi Kong, Noah Constant, Petr Pilar, Heming Ge, Yun-Hsuan Sung, Brian Strope, and Ray Kurzweil. 2018. Learning semantic textual similarity from conversations. In Proceedings of The Third Workshop on Representation Learning for NLP, pages 164-174, Melbourne, Australia. Association for Computational Linguistics.

Saizheng Zhang, Emily Dinan, Jack Urbanek, Arthur Szlam, Douwe Kiela, and Jason Weston. 2018. Personalizing dialogue agents: I have a dog, do you have pets too? In Proceedings of the 56th Annual Meeting of the Association for Computational Linguistics (Volume 1: Long Papers), pages 2204 2213, Melbourne, Australia. Association for Computational Linguistics. 


\section{A Additional Results}

\begin{tabular}{|c|c|c|c|c|c|c|c|c|c|c|c|c|}
\hline & \multicolumn{6}{|c|}{$\mathrm{MT}+\mathrm{FT}$} & \multicolumn{6}{|c|}{ All Tasks MT } \\
\hline & \multirow[t]{2}{*}{ PPL } & BLEU & \multicolumn{3}{|c|}{ ROUGE } & \multirow[t]{2}{*}{$\mathrm{F} 1$} & \multirow[t]{2}{*}{ PPL } & BLEU & \multicolumn{3}{|c|}{ ROUGE } & F1 \\
\hline & & & 1 & 2 & $\mathrm{~L}$ & & & 4 & 1 & 2 & $\mathrm{~L}$ & \\
\hline ConvAI2 & 11.1 & 6.6 & 37.0 & 11.6 & 31.8 & 21.6 & 10.8 & 5.5 & 39.4 & 12.5 & 33.7 & 21.7 \\
\hline DailyDialog & 10.4 & 4.0 & 35.6 & 10.0 & 30.8 & 18.2 & 12.0 & 2.9 & 33.9 & 8.7 & 29.2 & 16.2 \\
\hline Wiz. of Wikipedia & 8.3 & 21.5 & 55.3 & 28.4 & 44.9 & 38.4 & 8.4 & 21.0 & 53.2 & 28.0 & 45.4 & 38.4 \\
\hline Empathetic Dialog & 11.4 & 3.5 & 38.0 & 9.5 & 32.3 & 19.5 & 11.5 & 3.7 & 37.2 & 8.9 & 31.4 & 19.3 \\
\hline Cornell Movie & 20.2 & 2.5 & 29.5 & 6.7 & 25.7 & 12.4 & 22.2 & 2.1 & 29.1 & 6.5 & 25.6 & 11.9 \\
\hline LIGHT & 18.9 & 2.6 & 30.8 & 5.8 & 24.8 & 16.2 & 19.3 & 2.4 & 30.5 & 5.6 & 24.6 & 16.1 \\
\hline ELI5 & 21.0 & 3.7 & 38.6 & 7.2 & 22.1 & 23.1 & 24.9 & 3.2 & 35.2 & 6.3 & 20.5 & 21.3 \\
\hline Ubuntu & 17.1 & 2.5 & 27.0 & 5.0 & 22.8 & 12.7 & 23.1 & 3.7 & 26.0 & 4.3 & 22.0 & 12.1 \\
\hline Twitter & 30.7 & 3.2 & 16.5 & 3.3 & 14.3 & 9.9 & 38.2 & 2.6 & 19.4 & 3.3 & 16.5 & 9.8 \\
\hline pushshift.io Reddit & 25.6 & 2.1 & 24.1 & 4.5 & 18.7 & 13.6 & 27.8 & 1.6 & 23.4 & 4.2 & 18.1 & 13.5 \\
\hline Image Chat & 18.8 & 2.4 & 30.1 & 5.7 & 26.0 & 13.0 & 22.3 & 2.1 & 28.4 & 4.9 & 24.6 & 12.9 \\
\hline IGC & 11.9 & 8.6 & 65.0 & 34.1 & 60.5 & 38.4 & 12.0 & 8.0 & 61.3 & 28.3 & 56.8 & 41.4 \\
\hline dodecaScore & 17.1 & 5.3 & 35.6 & 11.0 & 29.6 & 19.8 & 19.4 & 4.9 & 34.8 & 10.1 & 29.0 & 19.6 \\
\hline
\end{tabular}

Table 8: Test performance for various metrics on the dodecaDialogue tasks comparing our multi-task and multitask + fine-tuned methods.

\begin{tabular}{|c|c|c|c|c|c|c|c|c|c|c|c|c|}
\hline & \multicolumn{6}{|c|}{$\mathrm{MT}+\mathrm{FT}$} & \multicolumn{6}{|c|}{ All Tasks MT } \\
\hline & \multirow[t]{2}{*}{ PPL } & BLEU & \multicolumn{3}{|c|}{ ROUGE } & \multirow[t]{2}{*}{$\mathrm{F} 1$} & \multirow[t]{2}{*}{ PPL } & BLEU & \multicolumn{3}{|c|}{ ROUGE } & F1 \\
\hline & & & 1 & 2 & $\mathrm{~L}$ & & & 4 & 1 & 2 & $\mathrm{~L}$ & \\
\hline ConvAI2 & 11.2 & 5.7 & 36.7 & 10.9 & 31.6 & 21.1 & 11.3 & 5.3 & 38.7 & 11.6 & 32.9 & 21.3 \\
\hline DailyDialog & 10.2 & 4.4 & 36.8 & 10.7 & 32 & 18.8 & 11.8 & 3.1 & 34.8 & 9.3 & 30.2 & 17.1 \\
\hline Wiz. of Wikipedia & 8.5 & 20.8 & 54.9 & 28.0 & 44.8 & 37.9 & 8.7 & 20.2 & 55.2 & 28.2 & 45.0 & 37.9 \\
\hline Empathetic Dialog & 11.1 & 3.6 & 38.6 & 9.8 & 32.7 & 19.7 & 11.2 & 3.5 & 37.5 & 9.1 & 31.8 & 19.3 \\
\hline Cornell Movie & 19.8 & 2.5 & 29.3 & 6.7 & 25.6 & 12.3 & 21.9 & 2.1 & 29.0 & 6.5 & 25.6 & 11.8 \\
\hline LIGHT & 18.7 & 2.6 & 31.2 & 6.2 & 25.2 & 16.5 & 19.0 & 2.5 & 30.9 & 6.1 & 25.0 & 16.4 \\
\hline ELI5 & 21.1 & 3.7 & 38.7 & 7.3 & 22.1 & 23.2 & 25.0 & 3.2 & 35.3 & 6.3 & 20.6 & 21.2 \\
\hline Ubuntu & 17.2 & 2.4 & 27.1 & 5.0 & 22.9 & 12.8 & 23.3 & 3.5 & 26.4 & 4.6 & 22.3 & 12.2 \\
\hline Twitter & 29.8 & 3.2 & 16.7 & 3.5 & 14.5 & 10.1 & 37.0 & 2.6 & 19.7 & 3.6 & 16.8 & 9.9 \\
\hline pushshift.io Reddit & 25.8 & 2.2 & 24.2 & 4.5 & 18.7 & 13.4 & 28.0 & 1.7 & 23.4 & 4.1 & 18.2 & 13.3 \\
\hline Image Chat & 18.3 & 2.4 & 30.7 & 6.2 & 26.3 & 14.3 & 21.8 & 2.1 & 28.6 & 5.3 & 24.7 & 13.1 \\
\hline IGC & 10.0 & 10.6 & 67.9 & 38.2 & 64.5 & 45.1 & 10.2 & 11.0 & 66.3 & 34.8 & 61.4 & 45.3 \\
\hline dodecaScore & 16.8 & 5.3 & 36.1 & 11.4 & 30.1 & 20.4 & 19.1 & 5.1 & 35.5 & 10.8 & 29.5 & 19.9 \\
\hline
\end{tabular}

Table 9: Validation performance for various metrics on the dodecaDialogue tasks comparing our multi-task and multi-task + fine-tuned methods.

\begin{tabular}{l|rrrrrr} 
& PPL & BLEU & \multicolumn{1}{c}{ ROUGE } & f1 \\
ConvAI2 & & 4 & 1 & 2 & $\mathrm{~L}$ & \\
DailyDialog & 11.3 & 5.6 & 22.2 & 7.0 & 20.4 & 21.3 \\
Wiz. of Wikipedia & 11.8 & 4.8 & 18.9 & 5.6 & 17.6 & 16.6 \\
Empathetic Dialog & 8.7 & 19.7 & 40.9 & 22.6 & 36.9 & 37.7 \\
Cornell Movie & 11.2 & 4.8 & 20.9 & 5.6 & 19.0 & 19.3 \\
LIGHT & 21.9 & 3.3 & 14.2 & 3.2 & 13.4 & 11.3 \\
ELI5 & 19.0 & 2.9 & 17.0 & 3.4 & 15.0 & 16.2 \\
Ubuntu & 25.0 & 1.6 & 14.2 & 2.6 & 9.6 & 16.2 \\
Twitter & 23.3 & 2.3 & 12.5 & 1.9 & 11.6 & 11.2 \\
pushshift.io Reddit & 37.0 & 2.3 & 9.5 & 1.7 & 8.7 & 8.9 \\
Image Chat (all turns) & 28.0 & 1.8 & 12.1 & 2.2 & 10.4 & 11.3 \\
IGC & 21.8 & 2.1 & 14.7 & 2.5 & 13.6 & 13.1 \\
\hline dodecaScore & 10.2 & 5.5 & 50.7 & 25.3 & 49.1 & 36.0 \\
\hline
\end{tabular}

Table 10: All Tasks Multi-Tasking (MT) validation performance for various metrics on the dodecaDialogue tasks with one set of decoding parameters: a beam size of 3 , minimum response length of 10 , and blocking repeated tri-grams. 


\begin{tabular}{|c|c|c|c|c|c|c|c|c|c|c|c|c|c|c|c|}
\hline \multirow[b]{3}{*}{ ConvAI2 } & \multicolumn{5}{|c|}{ BLEU } & \multicolumn{5}{|c|}{ ROUGE-L } & \multicolumn{5}{|c|}{$\mathrm{F} 1$} \\
\hline & Score & Beam & $\operatorname{Min} \mathrm{L}$ & $\operatorname{Max} \mathrm{L}$ & N-gram Block & Score & Beam & $\operatorname{Min} L$ & $\operatorname{Max} \mathrm{L}$ & N-gram Block & Score & Beam & $\operatorname{Min} \mathrm{L}$ & $\operatorname{Max} \mathrm{L}$ & N-gram Block \\
\hline & 5.7 & 10 & 10 & 128 & 3 & 31.6 & 10 & 50 & 128 & 3 & 21.1 & 3 & 10 & 128 & 3 \\
\hline DailyDialog & 4.4 & 10 & 5 & 128 & 3 & 32.0 & 3 & 50 & 128 & 3 & 18.8 & 5 & 10 & 128 & 3 \\
\hline Wiz. of Wikipedia & 20.8 & 10 & 5 & 128 & 0 & 44.8 & 10 & 50 & 128 & 3 & 37.9 & 10 & 10 & 128 & 3 \\
\hline Empathetic Dialog & 3.6 & 10 & 5 & 128 & 3 & 32.7 & 5 & 50 & 128 & 3 & 19.7 & 5 & 10 & 128 & 3 \\
\hline Cornell Movie & 2.5 & 10 & 5 & 128 & 3 & 25.6 & 10 & 50 & 128 & 3 & 12.3 & 10 & 20 & 128 & 3 \\
\hline LIGHT & 2.6 & 3 & 5 & 128 & 3 & 25.2 & 5 & 50 & 128 & 3 & 16.5 & 5 & 20 & 128 & 3 \\
\hline ELI5 & 3.7 & 10 & 200 & 256 & 3 & 22.1 & 5 & 200 & 256 & 3 & 23.2 & 10 & 200 & 256 & 3 \\
\hline Ubuntu & 2.4 & 10 & 5 & 128 & 0 & 22.9 & 10 & 40 & 128 & 3 & 12.8 & 2 & 10 & 128 & 3 \\
\hline Twitter & 3.2 & 10 & 20 & 128 & 3 & 14.5 & 5 & 50 & 128 & 3 & 10.1 & 10 & 20 & 128 & 3 \\
\hline pushshift.io Reddi & 2.2 & 10 & 10 & 128 & 0 & 18.7 & 5 & 50 & 128 & 3 & 13.4 & 5 & 50 & 128 & 3 \\
\hline Image Chat (all turns) & 2.4 & 10 & 5 & 128 & 3 & 26.4 & 3 & 50 & 128 & 3 & 14.3 & 5 & 1 & 128 & 3 \\
\hline IGC & 10.6 & 10 & 5 & 128 & 3 & 64.5 & 3 & 50 & 128 & 3 & 45.1 & 10 & 5 & 128 & 3 \\
\hline
\end{tabular}

Table 11: Best decoding parameters for each task, based on metric. Scores are from the best performing taskspecific multi-task + fine-tuned model on validation sets. "Min L" and "Max L" refer to the minimum and maximum decoding length, where "L" is the number of tokens.

\section{B Human Evaluation Further Details}

We provide additional results from our human evaluations described in Section 5. In Figure 1, we compare our All Tasks MT Image+Seq2Seq model to existing baselines from both tasks; to produce those outputs, we used beam search with a beam size of 10 and tri-gram blocking. As with our experiments regarding automatic metrics, we additionally explored nucleus sampling, with parameter $p=0.7$, and compared to both the baseline models as well as human outputs. In tables 12, 13, and 14, we show the full results of comparing various models both to each other and also to humans.

When collecting the model-human chats for Wizard of Wikipedia, we additionally asked the humans for a rating from 1-5 at the end of each conversation, to indicate the quality of the model's responses; we compare these Likert ratings to that of Dinan et al. (2019), which followed the same protocol, in Table 15. The findings are similar to the pairwise ACUTE-Eval results in the main paper.

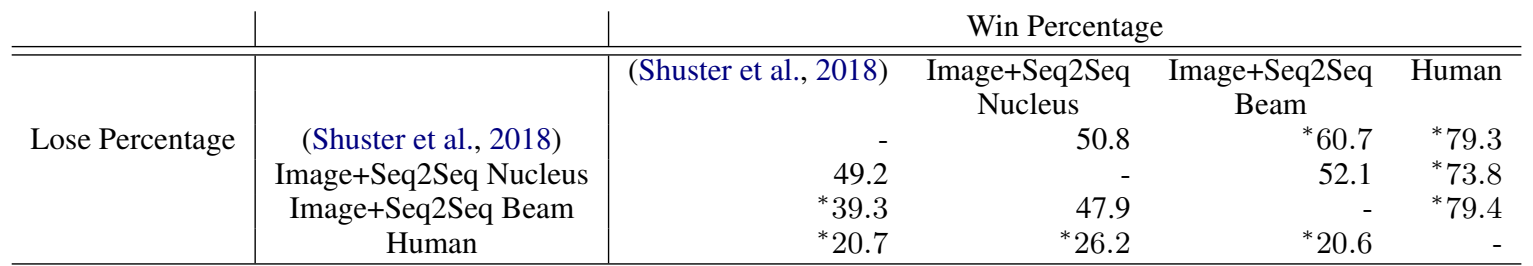

Table 12: Human evaluations on Image Chat, comparing various decoding schemes for our Image+Seq2Seq model trained on all tasks MT, as well as comparisons with human outputs. Scores with * are statistically significant (binomial test, $p<.05$ ).

\begin{tabular}{|c|c|c|c|c|c|}
\hline & & \multicolumn{4}{|c|}{ Win Percentage } \\
\hline \multirow{5}{*}{ Lose Percentage } & & (Dinan et al., 2019) & $\begin{array}{c}\text { Image+Seq2Seq } \\
\text { Nucleus }\end{array}$ & $\begin{array}{c}\text { Image+Seq2Seq } \\
\text { Beam }\end{array}$ & Human \\
\hline & (Dinan et al., 2019) & - & 59.1 & 62.1 & 71.9 \\
\hline & Image + Seq 2 Seq Nucleus & 40.1 & - & - & 70.4 \\
\hline & Image+Seq2Seq Beam & 37.9 & - & - & 60.0 \\
\hline & Human & 28.1 & 29.6 & 40.0 & - \\
\hline
\end{tabular}

Table 13: Human evaluations on Wizard of Wikipedia (seen) test set, comparing various decoding schemes for our Image+Seq2Seq model trained on all tasks MT, as well as comparisons with human outputs, using ACUTE-Eval. All scores are statistically significant (binomial test, $p<.05$ ). 


\begin{tabular}{c|c|rrrr} 
& & \multicolumn{4}{|c}{ Win Percentage } \\
\hline \hline \multirow{5}{*}{ Lose Percentage } & (Dinan et al., 2019) & \multicolumn{2}{c}{ Image+Seq2Seq } & Image+Seq2Seq & Human \\
& & & Nucleus & Beam & \\
& (Dinan et al., 2019) & - & 62.3 & 64.1 & 75.8 \\
& Image+Seq2Seq Nucleus & 37.7 & - & - & 72.8 \\
& Image+Seq2Seq Beam & 35.9 & - & - & 60.5 \\
& Human & 24.2 & 27.2 & 39.5 & - \\
\hline
\end{tabular}

Table 14: Human evaluations on Wizard of Wikipedia (unseen) test set, comparing various decoding schemes for our Image+Seq2Seq model trained on all tasks MT, as well as comparisons with human outputs, using ACUTEEval. All scores are statistically significant (binomial test, $p<.05$ ).

\begin{tabular}{llrr}
\hline Method & Decode Method & Seen & Unseen \\
\hline Human Performance & & $4.13(1.08)$ & $4.34(0.98)$ \\
(Dinan et al., 2019) & Beam & $2.92(1.33)$ & $2.93(1.30)$ \\
Image+Seq2Seq (All Tasks MT) & Beam & $3.42(1.10)$ & $3.33(1.09)$ \\
Image+Seq2Seq (All Tasks MT) & Nucleus & $2.95(1.08)$ & $3.43(1.05)$ \\
\hline
\end{tabular}

Table 15: Human evaluations on Wizard of Wikipedia, comparing various decoding schemes for our Image+Seq2Seq model trained on all tasks MT, as well as comparisons with human outputs, in terms of Likert Scores. Ratings are reported as mean (stddev). 


\section{All Tasks Multi-Task Model Examples}

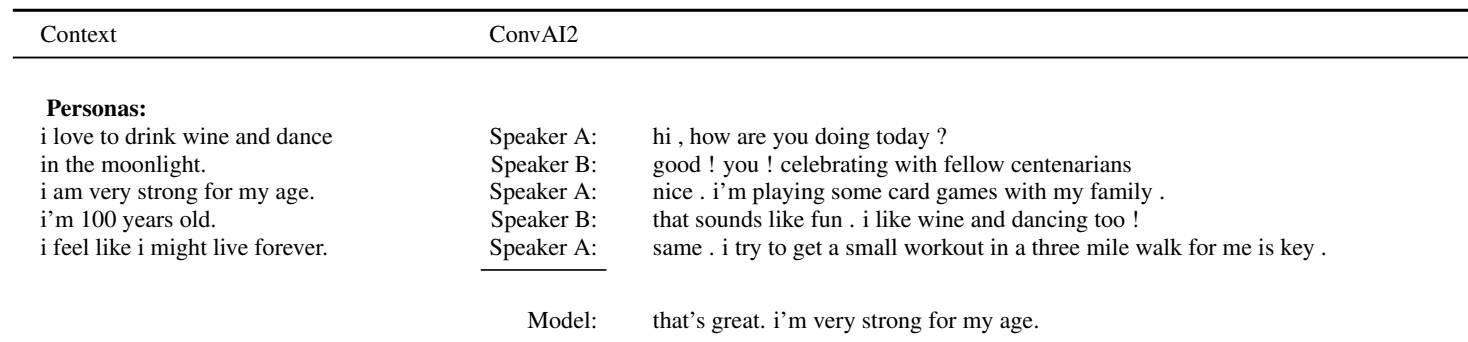

\begin{tabular}{|c|c|c|}
\hline Context & \multicolumn{2}{|c|}{ Wizard of Wikipedia } \\
\hline \multirow{5}{*}{$\begin{array}{l}\text { Chosen Topic: } \\
\text { The Rolling Stones } \\
\text { Knowledge: } \\
\text { no_passages_used }\end{array}$} & & \\
\hline & Speaker A: & I love the Rolling Stones. Do you? They were formed in 1962. \\
\hline & Speaker B: & I love them! Who was in the band at the start? \\
\hline & Speaker A: & $\begin{array}{l}\text { The first lineup was Brian Jones, Mick, Keith, Bill Wyman, Charlie Watts and Ian } \\
\text { Steward. }\end{array}$ \\
\hline & Speaker B: & Was Mick ever replaced? \\
\hline
\end{tabular}

Context Wizard of Wikipedia

Chosen Topic:

Dog

Knowledge:

The dog was the first species to be domesticated and has been selectively

bred over millennia for various

behaviors, sensory capabilities, and

physical attributes.
Speaker A: Hi, do you like dogs?

Speaker B: I do! Actually, my favourite is the Basenji breed! It is a hunting dog.

Speaker A: Oh, those are cool. I have seen them on the Westminster Dog Show, but I've never met one in person. I have a Havanese.

Speaker B: Wow, are you Cuban? I know that the Havanese are the national dog of cuba!

Speaker A: No, not Cuban, but yes they are the national dog of Cuba! Apparently all the Havanese in the US are descended from a handful that came here from Cuba during the revolution!

Model: that's so cool! the dog was the first species to be domesticated

\begin{tabular}{|c|c|c|}
\hline Context & \multicolumn{2}{|c|}{ Wizard of Wikipedia } \\
\hline $\begin{array}{l}\text { Chosen Topic: } \\
\text { Orc } \\
\text { Knowledge: } \\
\text { While the overall concept of orcs draws } \\
\text { on a variety of pre-existing mythology, } \\
\text { the main conception of the creatures } \\
\text { stems from the fantasy writings of } \\
\text { J. R. R. Tolkien, in particular "The Lord } \\
\text { of the Rings". }\end{array}$ & $\begin{array}{l}\text { Speaker A: } \\
\text { Speaker B: } \\
\text { Speaker A: } \\
\text { Speaker B: }\end{array}$ & $\begin{array}{l}\text { Orcs are cool fictional humanoid beings } \\
\text { Yeah I've seen them in a lot of things like Magic and DnD. } \\
\text { While the concept remains the same it draws on many preexisting mythology } \\
\text { That's really interesting! Orcs are some of my favorite fictional characters hon- } \\
\text { estly. } \\
\text { the main conception of the creatures stems from the fantasy writings of the lord } \\
\text { of the rings }\end{array}$ \\
\hline
\end{tabular}




\begin{tabular}{|c|c|c|}
\hline Context & \multicolumn{2}{|c|}{ Empathetic Dialogues } \\
\hline $\begin{array}{l}\text { Situation } \\
\text { I received concert tickets for Christmas. } \\
\text { Emotion } \\
\text { excited }\end{array}$ & $\begin{array}{l}\text { Speaker A: } \\
\text { Speaker B: } \\
\text { Speaker A: }\end{array}$ & $\begin{array}{l}\text { I couldn't wait to go to the concert. } \\
\text { Which Concert? } \\
\text { The U2 concert. Tickets were really expensive and I never thought we would be } \\
\text { able to go, but somehow we did!!! }\end{array}$ \\
\hline
\end{tabular}

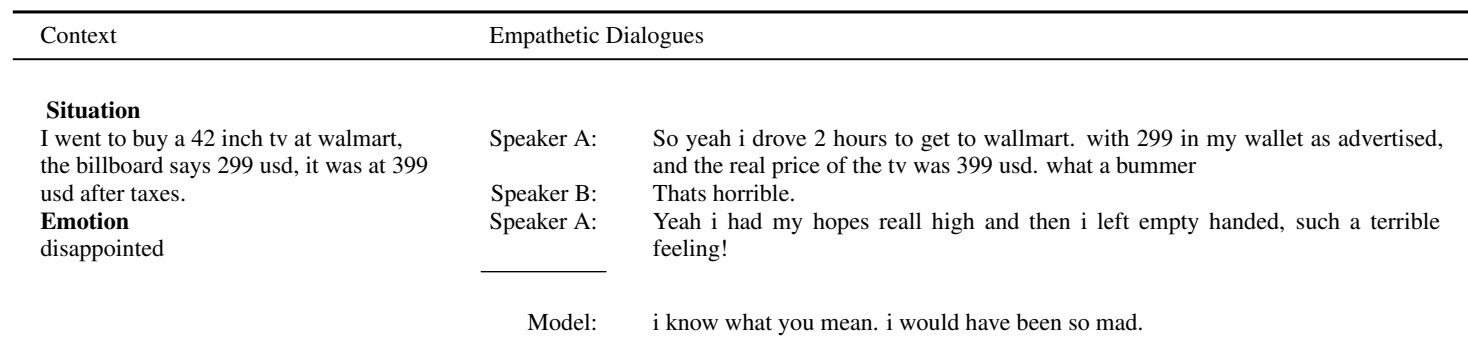

\begin{tabular}{|c|c|c|}
\hline Context & \multicolumn{2}{|c|}{ Empathetic Dialogues } \\
\hline Situation & Speaker A: & hi, i feel so lonely sometimes because all my friends live in a different country \\
\hline $\begin{array}{l}\text { All my friends live in a different country } \\
\text { Emotion }\end{array}$ & Speaker B: & $\begin{array}{l}\text { Oh, I'm sure you are lonely. Maybe you can join some kind of club that lets you } \\
\text { meet new friends? }\end{array}$ \\
\hline lonely & Speaker A: & i was thinking about it! I wanted to join a group for local moms \\
\hline
\end{tabular}

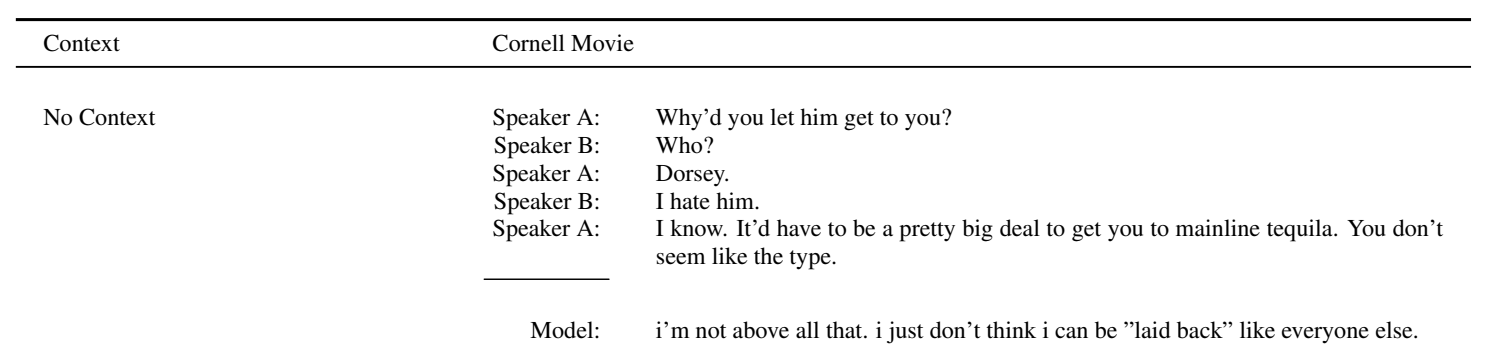

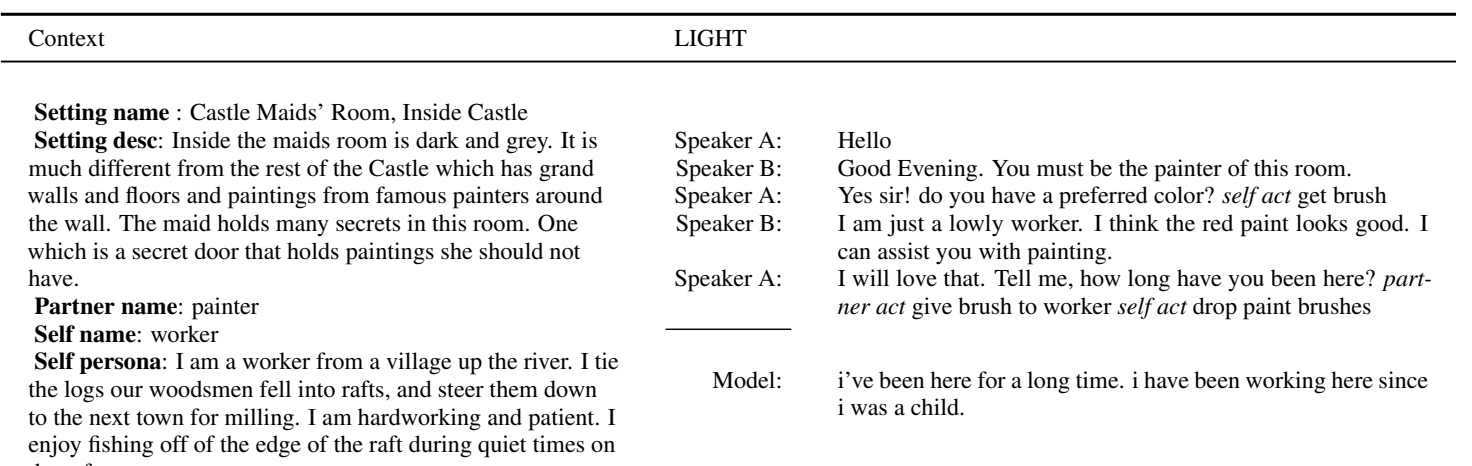




\begin{tabular}{lll}
\hline Context & Daily Dialog \\
\hline Topic & \\
ordinary_life & Speaker A: & So how did I do on my driving test ? \\
Emotion & Speaker B: & Do you want the truth ? \\
no_emotion & Speaker A: & Of course, I do . \\
& Speaker B: & Well , you really didn't do all that well . \\
& Speaker A: & How bad did I do ? \\
\cline { 2 - 2 } & Model: & don't worry, i'll give you another try.
\end{tabular}

\begin{tabular}{|c|c|c|}
\hline Context & \multicolumn{2}{|c|}{ pushshift.io Reddit } \\
\hline \multirow[t]{2}{*}{$\begin{array}{l}\text { Subreddit } \\
\text { beer }\end{array}$} & Speaker A: & $\begin{array}{l}\text { In Santa Cruz? Be SURE to hit up Sante Adairius Rustic Ales in Capitola! If } \\
\text { you like farmhouse, funky, sour ales, they are real tough to beat. Their IPAs and } \\
\text { porters are also amazing. Another great new brewery is Discretion, in Capitola } \\
\text { and I am also a fan of Santa Cruz Mountain Brewing and Seabright Brewing for } \\
\text { affordability's sake ( } \$ 5 \text { pitchers at Seabright on Tuesday/Thursday I believe?) but } \\
\text { some beer snobs would turn their noses up at those places. Also, burger in Santa } \\
\text { Cruz and Aptos always has a great taplist, as well as Aptos BBQ in Aptos. }\end{array}$ \\
\hline & Model: & $\begin{array}{l}\text { thanks for the suggestions! i'll have to check out sante adairius rustic ales. i've } \\
\text { heard good things about seabright brewing, but haven't had a chance to try it yet } \\
\text { do you know much about it? }\end{array}$ \\
\hline
\end{tabular}

\begin{tabular}{ll}
\hline Context & Twitter \\
\hline No Context & $\frac{\text { Speaker A: }}{n}$ Model: $\quad$ reading now . this is absolutely fantastic . \\
& thank you so much. i'm glad you like it. i hope you enjoy it as much as i do.
\end{tabular}

\begin{tabular}{|c|c|c|}
\hline Context & Ubuntu & \\
\hline \multirow[t]{3}{*}{ No Context } & Speaker A: & $\begin{array}{l}\text { i set up my hd such that i have to type a passphrase to access it at boot. how can } \\
\text { i remove that passwrd, and just boot up normal. i did this at install, it works fine, } \\
\text { just tired of having reboots where i need to be at terminal to type passwd in. help? }\end{array}$ \\
\hline & Speaker B: & $\begin{array}{l}\text { backup your data, and re-install without encryption "might" be the easiest method } \\
\text {. }\end{array}$ \\
\hline & Model: & i'll try that, but i'm not sure how to do it without encryption.. \\
\hline
\end{tabular}

Knowledge

$<\mathrm{P}>$ And it does n't come without questioning and critical thought. What we feel entitled to is a better world . And the thing is, we can see it clearer and with broader scope than anyone before us, because all we know is the entire world, in real time. $<\mathrm{P}>$ Page 1 of 212 Next $>$ Spycke83 PSLS Level : Bronze Messages : 52 Likes Received : 20 PSN : Spycke83 I'm wondering what you guys think of this newish trend of gamers feeling they are entitled certain things from a game...
Speaker A: Why do recent generations feel so entitled, like everyone owes them ? $-\mathrm{T}-$

Model: it's not that they feel entitled, but that they don't have enough money to pay for it. 


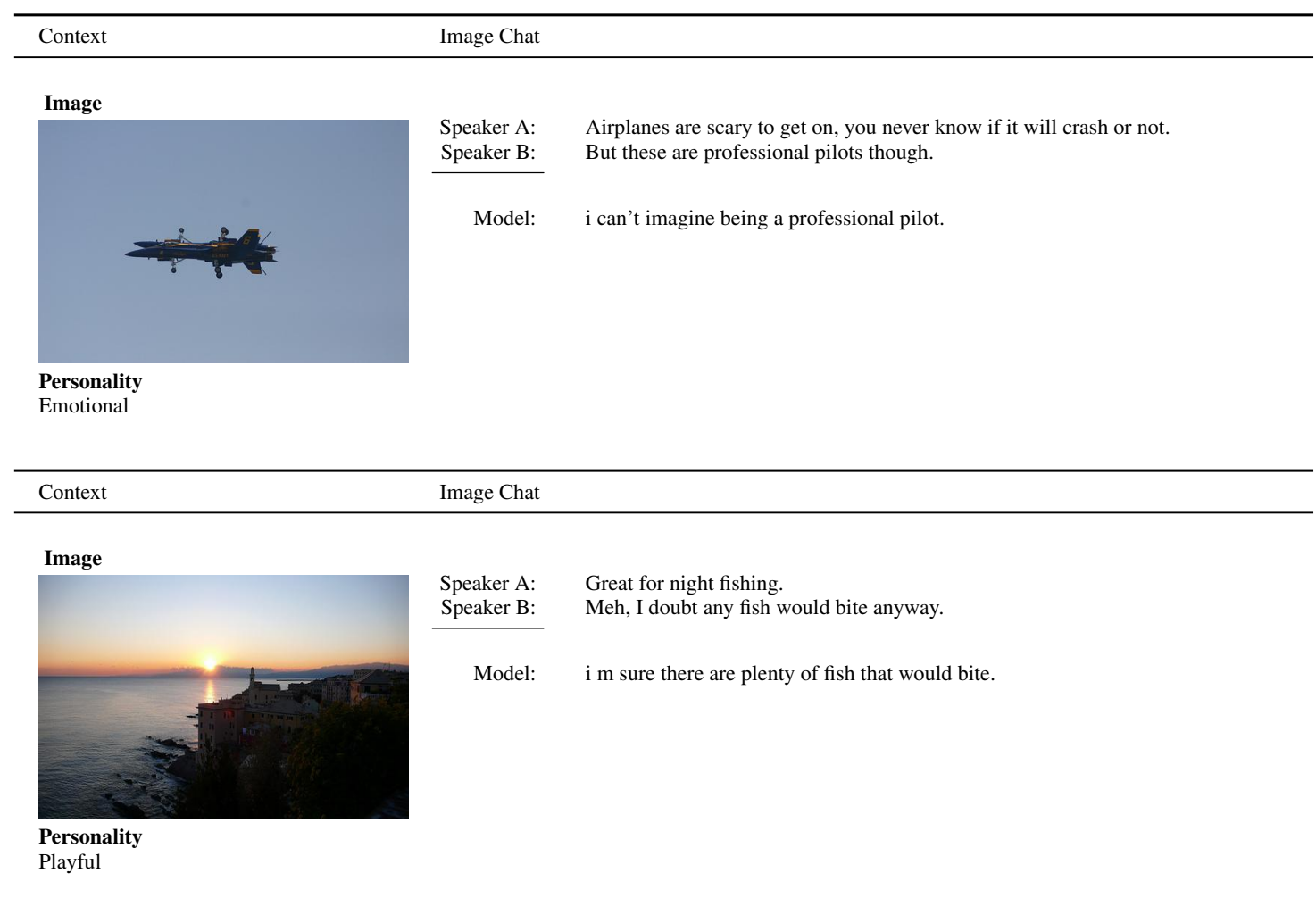

\begin{tabular}{l|l}
\hline Context & Image Chat (First Turn) \\
\hline Image & \\
\hline & \\
\hline $\begin{array}{l}\text { Personality } \\
\text { Curious }\end{array}$ \\
\hline Context & IGC \\
\hline
\end{tabular}

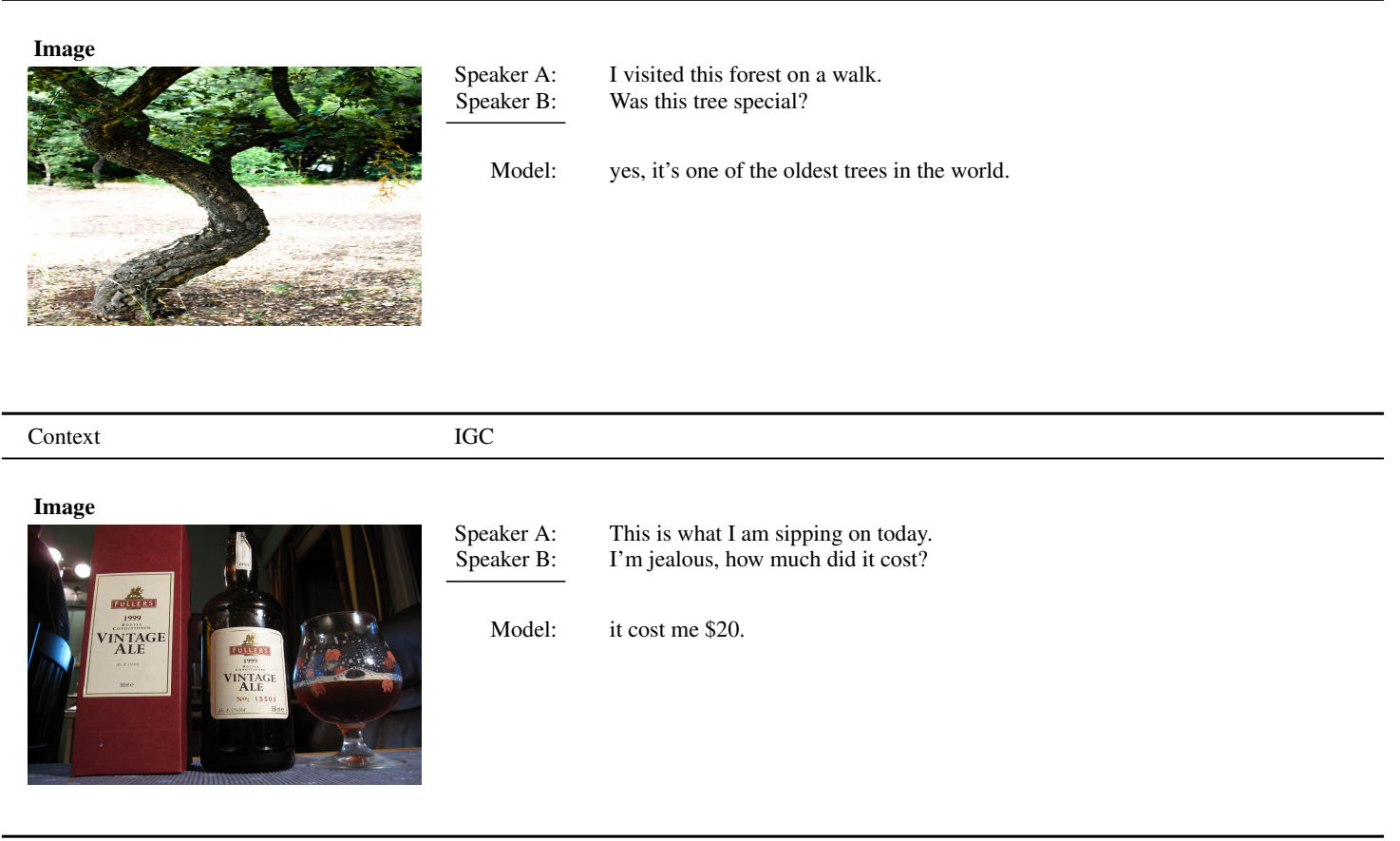

